\title{
Risk factors for non-cure among new sputum smear positive tuberculosis patients treated in tuberculosis dispensaries in Yunnan, China
}

\author{
Hua Jianzhao ${ }^{1 *}$, Susan van den Hof ${ }^{2,3}$, Xu Lin ${ }^{1}$, Qiu Yubang ${ }^{1}$, Hou Jinglong ${ }^{1}$ and Marieke J van der Werf ${ }^{2,3}$
}

\begin{abstract}
Background: Yunnan province in China has a high tuberculosis (TB) burden. Cure rates in general are high, but they were below the target of $85 \%$ in 26 out of 129 counties in 2005. In these 26 counties we assessed which patient-related and treatment-related factors were associated with non-cure.

Methods: We conducted a prospective cohort study. Smear positive pulmonary TB patients treated at the local Center for Disease Control and Prevention (CDC) were interviewed before start of treatment and during the fifth month of treatment using structured questionnaires. Information on treatment outcome was extracted from patient records. Patients cured at the end of treatment were compared to patients with unsuccessful treatment outcomes (failure, default, and death).
\end{abstract}

Results: A total of 841 patients were registered between January-June 2007 of which 792 (94\%) were cured. Independent risk factors for non-cure were having a low income (<3000 RMB per year), not having medical insurance, a delay in health care seeking $>30$ days, a positive smear test result two months after start of treatment, not being aware of the need to go to the CDC for medical follow up during treatment, and not seeing the need for treatment observation.

Conclusion: Reducing the financial burden of TB disease and providing health education to improve compliance with treatment could increase the proportion of patients with successful treatment outcomes.

\section{Background}

High quality tuberculosis (TB) treatment is a key factor of the Directly Observed Treatment Short-course (DOTS) strategy. In combination with a high case detection rate it is expected to reverse the TB epidemic. The targets for case detection and treatment success are $70 \%$ and $85 \%$ respectively [1]. Treatment success is defined as bacteriologically confirmed cure or a completed course of treatment without bacteriological proof of cure [2]. TB patients with an unsuccessful treatment outcome, especially failure and default, are a public health concern as these patients may have developed drug resistance and remain infectious for prolonged periods of time.

\footnotetext{
* Correspondence: ynhuajianzhao@hotmail.com

${ }^{1}$ Yunnan Provincial Centers for Disease Control and Prevention, Yunnan, China

Full list of author information is available at the end of the article
}

TB treatment outcomes are influenced by bacterium characteristics such as drug resistance, patient characteristics, patient behavior, and quality of health care [3-8]. Poor adherence to anti-tuberculosis treatment is considered the most important factor leading to non-curein patients infected with strains susceptible to the drugs [9-11]. Therefore, observation of treatment is considered one of the key elements of the DOTS strategy. It has been described before that in some places directly observed treatment (DOT) by village health workers was not very successful, due to insufficient financial incentives for the village health workers in relation to the task of thrice-weekly DOT for 6-8 months [12]. In remote areas, DOT can also be performed by an educated family member or community volunteer [2]. The influence of this approach on treatment outcomes is not known.

Yunnan province in southern China is a high TB burden province with poor economic status. The province

\section{Biomed Central}


covers $394,000 \mathrm{~km}^{2}$ of which $94 \%$ is mountainous area. Seventy-four percent of the 44 million inhabitants live in rural areas. Under China's National TB Control Program (NTP), Yunnan province has implemented the DOTS strategy since 2002. In 2004, based on estimates by the Ministry of Health, the provincial case detection rate reached $70 \%$ [13]. In 2005, the average treatment success rate was $92 \%$ and the average cure rate was $92 \%$. In 24 out of the 129 counties in Yunnan the treatment success rate was below $85 \%$ and in 26 counties the cure rate was below $85 \%$.

The Center for Disease Control and Prevention (CDC) of Yunnan province decided to assess which TB patientrelated and treatment-related factors are the main determinants for non-cure in the counties of Yunnan province in China with a relatively low cure rate. Therefore, we conducted a prospective cohort study in counties with a cure rate below $85 \%$ in 2005 . The results may be used to develop policies to improve treatment success in those areas.

\section{Methods}

\section{Setting, study design, and population}

A prospective cohort study was performed in these counties to identify factors that are associated with noncure. All new sputum smear-positive TB patients over 14 years of age registered between January and June 2007 by the local CDC in the 26 counties with a cure rate $<85 \%$ in 2005 were eligible for inclusion in our prospective cohort study. Informed consent was taken before inclusion in the study.

\section{Sample size}

For the sample size calculation we considered type of treatment observer as the main factor associated with treatment outcome. We assumed that $67 \%$ of the TB patients are observed by a family member and the remaining $33 \%$ are observed by a village doctor (ratio $2: 1$, based on expert opinion). We wanted to be able to show a difference in treatment success rate of $10 \%$ between the two groups $(85 \%$ in those observed by a family member and $95 \%$ in those observed by a village doctor). Taking into account a maximum of $15 \%$ missing data, a power of 0.8 and alpha of 0.05 , a total sample size of 744 patients was required.

\section{Data collection}

All eligible TB patients were approached by the local CDC doctors. TB patients who agreed to participate in the survey were interviewed before start of TB treatment and during the fifth month of treatment using structured questionnaires. In the first interview we collected information about patient characteristics, such as age, sex, educational background, TB symptoms, and co-morbidity (diabetes, HIV/AIDS, lung cancer, liver and kidney diseases, and other co-morbidity). Information on treatment adherence, side effects, patient's knowledge of TB treatment and perceived need for DOT was included in the second interview. At that time, treatment observers were also interviewed to collect information on direct observation of treatment and treatment interruption.

All TB patients were registered, treated and managed according to the procedures of the National Tuberculosis Control Plan. They all started standardized treatment directly after registration. The standardized treatment regimen consists of two months of thrice-weekly isonizid, rifampicin, pyrazinamide and ethambutol followed by four months of thrice-weekly isoniazid and rifampicin. Patient supervision and follow-up with sputum smear tests during and at the end of treatment was conducted as per the NTP guidelines.

Information on chest radiograph results and sputum smear microscopy results at month 2-3, month 5 and month 6 was extracted from the TB patient medical record at the local CDC. Treatment outcomes were registered at the end of treatment according to the WHO definitions [2].

Questionnaires were pre-tested, and modified according to the lessons learned in the pre-test. Directly after the interview the filled in questionnaires were checked for completeness and consistency. The researchers reinterviewed a random sample of $10 \%$ of the included individuals and cross checked data with other data sources. Rechecking showed satisfactory data quality.

\section{Operational definitions}

We used the definitions of the WHO for new smearpositive TB patient and treatment outcome [2]. Successful treatment includes cure and treatment completion, and unsuccessful treatment includes failure, death, and default. We also used the WHO definition for reporting of smear microscopy, where the reported result indicates the number of acid-fast bacilli observed. A result of at least 2 relates to at least 1 acid-fast bacillus observed per microscopy field [14].

Annual income was categorized into three groups of equal size: low income group $<3000$ Yuan (RMB); medium income group 3000-7000 Yuan (RMB); and high income group >7000 Yuan (RMB). One Yuan equalled about 0.13 USD in 2007.

According to China's TB Control Program (NTP) guidelines, anti-TB drugs should be taken every other morning (on even dates) before breakfast. Patients taking drugs at other times or intervals were considered as taking drugs not according to the guidelines.

Interruption of treatment means that patients missed at least one dose during treatment. Interruption of 
treatment was categorized as: (1) interruption of taking drugs during intensive phase, or (2) interruption of taking drugs during continuation phase, or (3) interruption of taking drugs during both phases.

\section{Data management and analysis}

Individual patient data were double entered by different persons in Epi data version 3.0 (Epidata Association, Denmark, 2006), and differences were checked. Patient data were analyzed using $\mathrm{R}$ software version 2.7.1 (R, New Zealand, 2008). A simple descriptive analysis was performed using percentages, means, medians, and standard deviations (SD). For the univariate analyses $\chi^{2}$ tests or Fisher's exact tests were used to compare the distribution of categorical variables between groups. The t-test was used to compare the distribution of age between participants and non-participants registered and treated by the TB dispensaries at the local CDCs.

Logistic regression analysis was conducted to identify risk factors for non-cure. Variables yielding a P-value $\leq 0.2$ in the univariate analysis were included in the multivariate analysis, and the model was refined by backward elimination guided by the change in log likelihood of successive models with a significance level of 0.05 .

\section{Ethical considerations}

The principles of the Helsinki Declaration were taken into account. Written, informed consent was obtained from all study subjects. The study was approved by the medical ethical committee of the Chinese TB Control Association.

\section{Results}

Of the 905 eligible TB patients registered between January and June 2007 in the 26 study counties, 59 (6.5\%) refused to participate, leaving 846 patients. Of the 846 patients interviewed before start of treatment, 833 were also interviewed during the fifth month of treatment. Eight could not be interviewed again as they had died, and five had defaulted and could not be traced. Of the TB patients who refused to participate, 35 (59.3\%) were male versus $576(68.1 \%)$ of the participating TB patients $(\mathrm{p}=0.28)$. Non participating patients were slightly older than participating patients (median age (SD), 39 (15.3) vs. 37 (15.7), $\mathrm{p}=0.11)$. Of the patients that refused to participate, 9 (15.0\%) had an unsuccessful treatment outcome.

The treatment success rate for the included smear positive pulmonary patients was 94\%; 792 (93.6\%) were cured and $5(0.6 \%)$ completed treatment. Out of the 49 patients with unsuccessful treatment outcome, 17 (2.0\%) failed treatment, $13(1.5 \%)$ stopped prematurely due to side effects, 10 (1.2\%) defaulted, and 9 (1.1\%) patients died. Those who completed treatment without bacteriological confirmation of cure were deleted from the analyses as it was not clear whether they were actually cured, leaving 841 patients for the study. Including these 5 patients in the group with successful treatment outcome did not affect the results significantly (data not shown).

Table 1 shows the characteristics of the participants. Most patients were male (68.4\%), under 30 years of age (33.9.\%), had no formal education beyond primary school $(62.6 \%)$, and had medical insurance $(81.8 \%)$. Seventhy-one percent of patients delayed seeking health care for TB symptoms for more than 30 days,. Almost all (98.0\%) had a sputum smear examination result after two months of treatment, and $11.7 \%$ of those were still sputum smear positive. Treatment was not observed for $27.7 \%$ of patients while for $49.3 \%$ of patients, treatment was observed by family members only. In $10.1 \%$ of cases, treatment was partly observed by health workers and partly by family members. Almost one in five patients (18.4\%) indicated to have interrupted treatment. During the fifth month of treatment, $9.0 \%$ of patients were unaware that during the treatment period he/she should go the CDC three times for medical follow-up including sputum smear examination; $38.4 \%$ did not know the correct treatment duration, and $74.7 \%$ did not perceive direct observation of treatment as necessary. A large proportion of those who reported to be unaware of the need for follow-up had interrupted treatment (87\% versus $22 \%, \mathrm{p}<0.001)$. Within this subgroup that had interrupted treatment and reported to be unaware of the need for follow-up, 52\% had missed up to seven doses in a row and $48 \%$ had missed more than seven doses consecutively.

In univariate analysis, patient characteristics significantly associated with non-cure were having no medical insurance, patient delay in seeking care for TB symptoms $>30$ days, and high initial sputum acid fast bacilli load $(>2+)$ (Table 1$)$. Treatment-related characteristics significantly associated with non-cure in univariate analysis were still having a positive sputum smear after two months of treatment, not having a DOT supervisor, and not taking drugs according to the NTP guideline. Also, patients who indicated that observation of treatment was not necessary, patients who were unaware of the need to go at least three times to the local CDC during treatment, and patients being unaware of the correct treatment duration were at increased risk of non-cure outcome.

Patient and treatment characteristics independently associated with non-cure were having no medical insurance, a low income, patient delay $>30$ days, a positive 2 -month smear test result, being unaware of the need to go to local CDC for check-ups during treatment, and refusal of direct observation of taking drugs. 
Table 1 Patient and treatment characteristics associated with non-cure in Yunnan province, China ( $\mathbf{N}=841$ )

Characteristic
Sex
Male
Female
Age (years)
$<30$
$\geq 30$
Education level**
No formal education or primary school only
Secondary school or higher
Medical insurance
No
Yes
Income***
Low
Middle
High
Co-morbidity
No
Yes
Patient delay (days)
$<30$
$\geq 30$
Directly observed treatment
Yes, by health care staff
Yes, shared by health care staff and family member
Yes, by family member
No
Sputum smear status before treatment
$\leq 2+$
$>2+$
$S p u t u m$ smear result after 2 months of treatment

Sputum smear result after 2 months of treatment

Negative

Positive

Without test

Adverse events**

No

Yes, did not require hospitalization

Yes, required hospitalization

Interruption of treatment

No

Yes, during intensive phase

Yes, during continued phase

Yes, during intensive and continuation phase

Maximum number of missed doses in a row ${ }^{* *}$

0

$1-7$

$>7$

Taking drugs according to guideline

Yes

No

$N(\%)$

575 (68.4)

$266(31.6)$

$285(33.9)$

$556(66.1)$

$520(62.6)$

$311(37.4)$

$153(18.2)$

$688(81.8)$

$314(37.3)$

$239(28.4)$

$288(34.3)$

$755(89.8)$

$86(10.2)$

$243(28.9)$

$598(71.1)$

108 (12.8)

$85(10.1)$

415 (49.3)

$233(27.7)$

$503(59.8)$

$338(40.2)$

non-cure (\%)

$726(86.3)$

$98(11.7)$

$17(2.0)$

539 (65.8)

$262(32.0)$

$18(2.2)$

686 (81.6)

28 (3.3)

96 (11.4)

31 (3.7)

682 (81.6)

102(12.2)

$52(6.2)$

$638(75.9)$

203 (24.1)
37 (6.4)

$12(4.5)$

$18(6.3)$

$31(5.6)$

$38(7.3)$

$10(3.2)$

15 (9.8)

$34(4.9)$

0.48

$0.32-0.90$

$21(6.7)$

$18(7.5)$

$10(3.5)$

$40(5.3)$

9 (11.6)

$7(2.9)$

$42(7.5)$

$4(3.7)$

9 (10.6)

$15(1.8)$

$21(9.0)$

$22(4.4)$

$27(8.0)$

$R R$

$95 \% \mathrm{Cl}$

$0.69 \quad 0.35-1.3$

1

0.88

$0.48-1.6$

$0.42 \quad 0.21-0.86$

0.02

0.06

0.02

0.01

0.03

0.01

$21(2.9) \quad 1$

11 (11.1)

17 (100)

$$
4.2
$$$$
34.8
$$

$2.0-9.1$

$24.7-48.6 \#$

0.06

$22(4.1)$

$10(3.8)$

$3(16.7)$

0.93

$$
4.7
$$

$0.44-2.0$

$1.3-17.4$

$$
39(5.7)
$$

$3(10.7)$

$3(3.1)$

4 (12.9)

$$
2.0
$$

0.54

$0.58-6.9$

$0.16-1.8$

$0.82-7.4$

0.09

$\begin{array}{rr}35(5.1) & 1 \\ 3(2.9) & 0.56 \\ 6(11.5) & 2.4\end{array}$

$0.17-1.9$

$0.97-6.0$

$<0.001$

19 (3.0)

30 (14.8)

\section{6}

0.01 02 11 
Table 1 Patient and treatment characteristics associated with non-cure in Yunnan province, $\mathrm{China}(\mathrm{N}=841)$ (Continued)

\begin{tabular}{|c|c|c|c|c|c|}
\hline $\begin{array}{l}\text { Patient knows that during treatment he/she should go to } \\
\text { CDC for medical check-ups** }\end{array}$ & & & & & $<0.001$ \\
\hline Yes & $752(91.0)$ & $22(2.9)$ & 1 & & \\
\hline No & $74(9.0)$ & $14(18.9)$ & 7.7 & $3.8-15.9$ & \\
\hline Patient knows the duration of TB treatment & & & & & 0.001 \\
\hline Yes & $518(61.6)$ & $19(3.7)$ & 1 & & \\
\hline No & $323(38.4)$ & $30(9.3)$ & 2.7 & $1.5-4.9$ & \\
\hline Patient agrees with the need for treatment observation ${ }^{* *}$ & & & & & 0.06 \\
\hline Yes & $209(25.3)$ & $4(1.9)$ & 1 & & \\
\hline No & $616(74.7)$ & $31(5.0)$ & 2.7 & $0.95-7.8$ & \\
\hline Family attitude ${ }^{* *}$ & & & & & 0.07 \\
\hline More supportive than usual & $339(40.3)$ & $9(2.7)$ & 1 & & \\
\hline No change or less supportive than usual & $502(59.7)$ & $40(8.0)$ & 2.1 & $0.95-4.5$ & \\
\hline
\end{tabular}

$\mathrm{RR}=$ Relative Risk, $95 \% \mathrm{Cl}=95 \%$ confidence interval.

Patients who reported to have interrupted treatment for less than two weeks less often were cured than patients who reported never to have missed a dose (Table 2).

\section{Discussion}

Many factors were observed to be related to non-cure in this study. Independent risk factors for non-cure of new smear-positive TB patients in the selected counties with a low cure rate in Yunnan province were: having no medical insurance; a low income, patient delay >30 days; a positive 2-month smear test result; being unaware of the need to go to local CDC for check-ups during treatment; and refusal of direct observation of drugs taking.

Almost one in ten patients $(9.0 \%)$ were unaware of the need to go to local CDC for medical check-ups including sputum smear examinations. These patients had an eight times higher risk of non-cure than patients who knew that they should attend medical check-ups at the CDC. More than one in ten patients $(11.7 \%)$ still had a positive sputum smear after two months of treatment, which is lower than the $18 \%$ observed in the registration data of Yunnan province in 2007 (unpublished data). Patients who were still sputum smear positive after two months had a six to seven times increased risk of noncure. Other independent risk factors for non-cure were not having medical insurance, a low income, patient delay in health care seeking for TB symptoms $>30$ days, not taking drugs according to the guideline, and not seeing the need for observation of treatment. Patients who indicated that they never had missed a dose more often were not cured than patients who indicated to have missed a maximum of 1-7 doses in a row. This is in contradiction with results of previous studies [4,15-17]. In these studies treatment interruption was associated with an increased risk of non-cure. The contradiction may partly be explained by the fact that many patients who died or failed treatment had not missed any dose according to the information provided: $92 \%$ versus 95\% among those cured. Furthermore, it may be that some patients that reported not to have missed a single dose might be afraid to admit that in fact they had missed some doses. Especially patients that did not respond well to treatment may be less likely to admit that they missed doses.

Patients with positive smears after 2 months of treatment may not react well to treatment due to an infection with drug resistant Mycobacterium tuberculosis, one of the major risk factors for non-cure. Drug susceptibility testing and treatment for multi-drug resistant tuberculosis were not implemented within the NTP in Yunnan province during the study, and no drug resistance survey has been conducted. We were unable to obtain information about the presence of drug resistance [18].

Patients with a DOT observer had a decreased risk of non-cure compared to patients without a DOT observer in univariate analysis, as has been observed in other studies [19-21]. Half of the patients had a family member as DOT observer and their outcomes were similar to the patients who had a health care worker as DOT observer. A recent study from Tanzania also observed similar treatment outcomes for sputum smear positive TB patients with community members, mostly family members, being DOT supervisors, compared to those with facility-based DOT [22].

Treatment was not observed at all for $28 \%$ of patients. DOT is the cornerstone of the DOTS strategy in China. In our data, lack of treatment observation was not an independent risk factor when adjusting for other factors influencing treatment outcome. This could be due to the fact that quality of DOT is not always sufficient, even if performed by health care workers like village doctors $[12,23]$. If it is not possible to increase the 
Table 2 Independent risk factors for non-cure in Yunnan province, China, identified by multivariate logistic regression analysis $\left(\mathrm{N}=\mathbf{8 1 3 ^ { \# }}\right)$

\begin{tabular}{|c|c|c|}
\hline Characteristic & OR $(95 \% \mathrm{Cl})$ & $\begin{array}{r}p- \\
\text { value }\end{array}$ \\
\hline Medical insurance & & 0.002 \\
\hline yes & 1 & \\
\hline no & $4.7(1.8-12.3)$ & \\
\hline Income* & & 0.05 \\
\hline low & 1 & \\
\hline middle & $0.45(0.16-1.3)$ & \\
\hline high & $0.22(0.06-0.82)$ & \\
\hline Patient delay (days) & & 0.25 \\
\hline$<30$ & 1 & \\
\hline$\geq 30$ & $6.3(1.3-32.0)$ & \\
\hline Sputum smear status before treatment & & 0.05 \\
\hline$\leq 2+$ & 1 & \\
\hline$>2+$ & $2.7(1.0-7.1)$ & \\
\hline $\begin{array}{l}\text { Sputum smear result after } 2 \text { months of } \\
\text { treatment }\end{array}$ & & $<0.001$ \\
\hline negative & 1 & \\
\hline positive & $6.0(2.3-15.9)$ & \\
\hline $\begin{array}{l}\text { Maximum number of missed } \\
\text { doses in a row }\end{array}$ & & 0.09 \\
\hline 0 & 1 & \\
\hline $1-7$ & $0.06(0.04-0.74)$ & \\
\hline$>7$ & $0.52(0.08-3.6)$ & \\
\hline Taking drugs according to guideline & & 0.03 \\
\hline yes & 1 & \\
\hline no & $2.8(1.1-7.1)$ & \\
\hline $\begin{array}{l}\text { Patient knows that during treatment } \\
\text { he/she should go to CDC for medical } \\
\text { check-ups }\end{array}$ & & 0.01 \\
\hline yes & 1 & \\
\hline no & $9.9(1.7-57.6)$ & \\
\hline $\begin{array}{l}\text { Patient agrees with the need for } \\
\text { treatment observation }\end{array}$ & & 0.05 \\
\hline yes & 1 & \\
\hline no & $4.4(0.98-20.1)$ & \\
\hline
\end{tabular}

OR = Odds Ratio, $95 \% \mathrm{Cl}=95 \%$ confidence interval.

* based on tertiles.

\# 841 patients in the study, but the questionnaire of 28 patients was not complete, so only 813 patients could be included in the multivariate model.

quality of DOT by village doctors, e.g. due to the high workload of village doctors, family members could provide DOT in China. A prerequisite is that they are trained well, and remain motivated and committed to the program.

Our study results support the results of previous reports that stated that delayed care seeking $(R R=2.7)$ and a high bacillary load at diagnosis (which may result from delayed care seeking) also increase the risk of noncure [4,16,24-27]. Public awareness of presence of TB dispensaries and the availability of free TB diagnosis and treatment is low in China. In a recent survey $42 \%$ of the interviewees were aware of the existence of TB dispensaries and $45 \%$ knew about the free diagnosis and treatment policy for TB [28].

Patients without medical insurance more frequently were not cured. In China, sputum smear examinations, chest radiographs and anti-TB drugs are provided free of charge, but additional costs, e.g. treatment of sideeffects are not $[29,30]$. Medical insurance covers part or all of these extra costs. The financial burden for those with no or partial insurance has been described to affect treatment adherence $[10,31]$. The financial burden is highest for those with a lower income. The 26 counties with a cure rate below $85 \%$ had a lower average gross domestic product per capita than the other counties in Yunnan province (11,980 versus $13,826 \mathrm{RMB})$. Also the number of health care workers per capita was lower (218 versus 244 per 100,000 inhabitants). These factors both may be an explanation for a lower overall cure rate.

Our study has a number of limitations. We included counties with a cure rate below $85 \%$ in 2005 in our study. During the study period in 2007, the average cure rate in these counties had increased to $94 \%$. The lower number of non-cured patients limited the statistical power to detect determinants of unsuccessful treatment. Furthermore, a lower successful treatment outcome percentage was observed among non-participants than among participants (15\% vs. $6 \%)$. Because the number of non-participants with unsuccessful treatment outcome was relatively small, we do not expect that non-participation in this study will have biased our findings to a relevant extent.

\section{Conclusions}

Our study has provided us with useful insights on factors influencing non-cure in Yunnan province. Based on the results, we recommend that patient education by clinic doctors should be enhanced to improve the patients' understanding of their disease and its treatment and to improve compliance with treatment and follow up. More resources and effort are needed to educate the general public to enable them to act effectively as community-based treatment observers.

\section{List of abbreviations used}

TB: tuberculosis; PTB: Pulmonary tuberculosis; NTP: China's TB Control Program; DOT: Observation of drug intake; DOTS: directly observed treatment short-course; CDC: Center for Disease Control and Prevention; SS+: Sputum smear positive; RR: relative risk; 95\%Cl: 95\% Confidence interval.

\section{Acknowledgements}

The authors are grateful to Foreign Loan Office of Ministry of Health of the People's Republic of China, Center for TB Control and Prevention (CDC) of China, Yunnan provincial health bureau and Yunnan CDC for providing the necessary support for the successful performance of the study. We 
appreciate the cooperation of all the doctors and tuberculosis patients participating in this study. The study was funded by the World Bank Loan and the UK Department for International Development.

\section{Author details}

${ }^{1}$ Yunnan Provincial Centers for Disease Control and Prevention, Yunnan, China. ${ }^{2}$ KNCV Tuberculosis Foundation, The Hague, The Netherlands. ${ }^{3}$ Center for Infection and Immunity Amsterdam (CINIMA), University of Amsterdam, Amsterdam, The Netherlands.

\section{Authors' contributions}

HJZ designed the study and wrote the manuscript. SH has been involved in monitoring of data-collection, data-analysis, and revision of the manuscript. $\mathrm{XL}, \mathrm{QYB}$ and HJL supervised data collection and were involved in dataanalysis. MJW has been involved in study design and revision of the manuscript. All authors have read the manuscript repeatedly and approved its final version.

\section{Competing interests}

The authors declare that they have no competing interests.

Received: 19 July 2010 Accepted: 11 May 2011 Published: 11 May 2011

\section{References}

1. Dye C, Gamett GP, Sleeman K, Williams BG: Prospects for worldwide tuberculosis control under the WHO DOTS strategy. Lancet 1998, 352:1886-91

2. World Health Organization: Treatment of Tuberculosis Guidelines for National Program. WHO/CDS/TB/2003.313. Third edition. Geneva, Switzerland: World Health Organization; 2003.

3. Pablos-Méndez A, Knirsch CA, Barr RG, Lerner BH, Frieden TR: Nonadherence in tuberculosis treatment: predictors and consequences in New York City. Am J Med 1997, 102:164-170.

4. Mangura B, Napolitano E, Passannante M, Sarrel M, McDonald R, Galanowsky K, Reichman L: Directly observed therapy (DOT) is not the entire answer: an operational cohort analysis. Int J Tuberc Lung Dis 2002, 6:654-661.

5. Santha T, Garg R, Frieden TR, Chandrasekaran V, Subramani R, Gopi PG, Selvakumar N, Ganapathy S, Charles N, Rajamma J, Narayanan PR: Risk factors associated with default, failure and death among tuberculosis patients treated in a DOTS program in Tiruvallur District, South India, 2000. Int J Tuberc Lung Dis 2002, 6:780-788.

6. Wares DF, Singh S, Acharya AK, Dangi R: Non-adherence to tuberculosis treatment in eastern Tarai of Nepal. Int J Tuberc Lung Dis 2003, 7:327-335.

7. Morsy AM, Zaher HH, Hassan MH, Shouman A: Predictors of treatment failure among tuberculosis patients under DOTS strategy in Egypt. East Mediterr Health J 2003, 9:689-701.

8. Hu D, Tan HZ, Quan J: Analysis of multiple factors of outcome in tuberculosis patients under chemotherapy. China Tropical Medicine 2004 4:712-713.

9. Caylà JA, Rodrigo T, Ruiz-Manzano J, Caminero JA, Vidal R, García JM, Blanquer R, Casals M, Working Group on Completion of Tuberculosis Treatment in Spain (Study ECUTTE): Tuberculosis treatment adherence and fatality in Spain. Respiratory Research 2009, 10:121.

10. Xu W, Lu W, Zhou Y, Shen H, Wang J: Adherence to anti-tuberculosis treatment among pulmonary tuberculosis patients: a qualitative and quantitative study. BMC Health Serv Res 2009, 9:169.

11. Widjanarko B, Gompelman M, Dijkers M, van der Werf MJ: Factors that influence treatment adherence of tuberculosis patients living in Java, Indonesia. Patient Prefer Adherence 2009, 3:231-238.

12. Tang S, Squire SB: What lessons can de drawn from tuberculosis Control in China in the 1990s? An analysis from a health system perspective. Health Policy 2005, 72:93-104.

13. National TB Control Program in 2005 work plan: Centers for Disease Control and Prevention, National Center for Tuberculosis Control, Beijing, China. 2005.

14. World Health Organization: Laboratory services in tuberculosis control. Part II: microscopy. WHO/TB/98.258 Geneva, Switzerland: World Health Organization; 1998.
15. Vasankari T, Holmström P, Ollgren J, et al: Risk factors for poor tuberculosis treatment outcome in Finland: a cohort study. BMC Public Health 2007, 7:291.

16. Bao QS, Du YH, Lu CY: Treatment outcome of new pulmonary tuberculosis in Guangzhou, China. BMC Public Health 2007, 7:344

17. Burman WJ, Cohn DL, Rietmeijer CA, Judson FN, Sbarbaro JA, Reves RR: Noncompliance With Directly Observed Therapy for Tuberculosis: Epidemiology and Effect on the Outcome of Treatment. Chest 1997, 111:1168-1173.

18. Churchyard GJ, Corbett EL, Kleinschmidt I, Mulder D, De Cock KM: Drugresistant tuberculosis in South African gold miners: incidence and associated factors. Int J Tuberc Lung Dis 2000, 4:433-440.

19. Balasubramanian VN, Oommen K, Samuel R: DOT or not? Direct observation of anti-tuberculosis treatment and patient outcomes, Kerala State, India. Int J Tuberc lung Dis 2000, 4:409-413.

20. Chee CB, Wang YT, Teleman MD, Boudville IC, Chew SK: Treatment outcome of Singapore residents with pulmonary tuberculosis in the first year after introduction of a computerized treatment surveillance module. Singapore Med J 2006, 47:529-533.

21. Balabanova Y, Drobniewski F, Fedorin I, Zakharova S, Nikolayevskyy V, Atun R, Coker R: The Directly Observed Therapy Short-Course (DOTS) strategy in Samara oblast, Russian Federation. Respir Research 2006, 7:44.

22. van den Boogaard J, Lyimo R, Irongo CF, Boeree MJ, Schaalma H, Aarnoutse RE, Kibiki GS: Community vs. facility-based directly observed treatment for tuberculosis in Tanzania's Kilimanjaro Region. Int I Tuberc Lung Dis 2009, 13:1524-1529.

23. Sun Q, Meng Q, Yip W, Yin X, Li H: DOT in rural China: experience from a case study in Shandong Province, China. Int J Tuberc Lung Dis 2008, 12:625-630.

24. Dewan PK, Arguin PM, Kiryanova H, Kondroshova NV, Khorosheva TM, Laserson K, Kluge H, Jakubowiak W, Wells C, Kazionny B: Risk factors for death during tuberculosis treatment in Orel, Russia. Int I Tuberc Lung Dis 2004, 8:598-602.

25. Farah MG, Tverdal A, Steen TW, Heldal E, Brantsaeter AB, Bjune G: Treatment outcome of new culture positive pulmonary tuberculosis in Norway. BMC Public Health 2005, 5:14

26. Gopi PG, Chandrasekaran V, Subramani R, Santha T, Thomas A, Selvakumar N, Narayanan PR: Association of conversion \& cure with initial smear grading among new smear positive pulmonary tuberculosis patients treated with Category I regimen. Indian I med Res 2006, 123:807-814.

27. Jakubowiak WM, Bogorodskaya EM, Borisov SE, Danilova ID, Kourbatova EV: Risk factors associated with default among new pulmonary TB patients and social support in six Russian regions. Int J Tuberc Lung Dis 2007, 11:46-53.

28. Lu SH, Tian BC, Kang XP, Zhang W, Meng XP, Zhang JB, Lo SK: Public awareness of tuberculosis in China: a national survey of 69253 subjects. Int J Tuberc Lung Dis 2009, 13:1493-1499.

29. Liu Q, Smith H, Wang Y, Tang S, Wang Q, Garner P: Tuberculosis patient expenditure on drugs and tests in subsidised, public services in China: a descriptive study. Tropical Medicine and International Health 2010, 15:26-32.

30. Hu D, Liu X, Chen J, Wang Y, Wang T, Zeng W, Smith H, Garner P: Direct observation and adherence to tuberculosis treatment in Chongqing, China: a descriptive study. Health Policy and Planning 2008, 23:43-55.

31. Liu X, Thomson R, Gong Y, Zhao F, Squire SB, Tolhurst R, Zhao X, Yan F, Tang S: How affordable are tuberculosis diagnosis and treatment in rural China? An analysis from community and tuberculosis patient perspectives. Tropical Medicine and International Health 2007, 12:1464-1471.

\section{Pre-publication history}

The pre-publication history for this paper can be accessed here: http://www.biomedcentral.com/1472-6963/11/97/prepub

\section{doi:10.1186/1472-6963-11-97}

Cite this article as: Jianzhao et al:: Risk factors for non-cure among new sputum smear positive tuberculosis patients treated in tuberculosis dispensaries in Yunnan, China. BMC Health Services Research 2011 11:97. 